\title{
NEUMANN SERIES OF BESSEL FUNCTIONS
}

BY

J. ERNEST WILKINS, JR.

1. Introduction. In this paper we shall study the possibility of representing a function $f(x)$ on $(0, \infty)$ by a Neumann series of the form

$$
\sum_{n=0}^{\infty} a_{n \nu} J_{\nu+2 n+1}(x),
$$

in which $\nu$ is an arbitrary real number. As a consequence of the identity [9, p. 404$]\left({ }^{1}\right)$

$$
\int_{0}^{\infty} t^{-1} J_{\lambda}(t) J_{\mu}(t) d t=2 \pi^{-1}\left(\lambda^{2}-\mu^{2}\right)^{-1} \sin 2^{-1}(\lambda-\mu) \pi \quad(\lambda+\mu>0),
$$

we conclude that the functions $(2 \nu+4 n+2)^{1 / 2} J_{\nu+2 n+1}(t)(n=0,1, \cdots ; \nu>-1)$ are orthonormal on the interval $(0, \infty)$ with weight function $t^{-1}$. Thus the coefficients $a_{n \nu}$ corresponding to a function $f(t)$ when $\nu>-1$ are

$$
a_{n \nu}=2(\nu+2 n+1) \int_{0}^{\infty} t^{-1} f(t) J_{\nu+2 n+1}(t) d t,
$$

and the Neumann series (1.1) assumes the form

$$
s_{\nu}(x)=\sum_{n=0}^{\infty} 2(\nu+2 n+1) J_{\nu+2 n+1}(x) \int_{0}^{\infty} t^{-1} f(t) J_{\nu+2 n+1}(t) d t .
$$

If $x^{-v} f(x)$, regarded as a function of a complex variable $x$, is regular and odd interior to a circle $(|x|=\rho)$, then it is known [9, pp. 524-525] that $f(x)$ admits an expansion of the form (1.1) which converges to $f(x)$ inside the circle $(|x|=\rho)$. Moreover, if $x^{-v} f(x)=\sum_{n=0}^{\infty} b_{n v} x^{2 n+1}$, then it is true that

$$
a_{n \nu}=2(\nu+2 n+1) \sum_{m=0}^{n} 2^{\nu+2 m} \Gamma(\nu+n+m+1) b_{m \nu} /(n-m) !
$$

The theory for functions of a real variable has been restricted in the past entirely to the case when $\nu=0$. The results of the earlier writers $[10,4,1]$ have been summarized in [9, pp. 533-535] where it is shown that when $f(t)$ is in $L(0, \infty), f(0+)$ exists and has the value zero( $\left.{ }^{2}\right)$, and the derivative $f^{\prime}(t)$

Presented to the Society, April 25, 1947; received by the editors August 15, 1947.

(1) Numbers in brackets refer to the bibliography at the end of the paper.

(2) This hypothesis is not explicitly stated by Watson. 
exists and is continuous on $(0, X)$, then the series $s_{0}(x)$ converges to $f(x)$ on $(0, X)$ if and only if the function

$$
F(t) \equiv f^{\prime}(t)-\frac{1}{2} \int_{0}^{\rightarrow \infty} v^{-1} J_{1}(v)\{f(t+v)-f(t-v)\} d v
$$

vanishes identically on $(0, X)$. Here $f(t)$ is defined for negative values of $t$ as $-f(-t)$. More recently Titchmarsh $[7, \mathrm{pp} .352-358]$ has shown that the same conclusion is valid provided that $f(t)\left(1+t^{3}\right)^{-1 / 2}$ is in $L(0, \infty), f(0+)$ exists and has the value zero, and the derivative exists and is continuous on $(0, X)$. He also investigated solutions of the equation $F(v) \equiv 0$.

For the case of an arbitrary real number $\nu$ we shall weaken Titchmarsh's integrability conditions and consider the class $\mathcal{A}_{\nu}$ of functions $f(t)$ possessing the properties that $t^{\nu} f(t) /(1+t)^{p+\nu+1 / 2}$ is in $L(0, \infty)$ for some nonnegative integer $p$ and that the limits

$$
\begin{aligned}
& \int_{a}^{\rightarrow \infty} t^{-3 / 2} f(t) \cos \left(t-\frac{1}{2} \nu \pi+\frac{1}{4} \pi\right) d t \\
& \int_{a}^{\rightarrow \infty} t^{-5 / 2} f(t) \sin \left(t-\frac{1}{2} \nu \pi+\frac{1}{4} \pi\right) d t
\end{aligned}
$$

exist when $a>0$. When $p=1$ and $\nu=0$ these conditions are equivalent to those of Titchmarsh. When $p=1$ the integrals (1.4) and (1.5) are absolutely convergent and when $p=2$ the integral (1.5) is. In order to make full use of our hypotheses we shall assume throughout the rest of the paper (as we may without any loss of generality) that $p \geqq 3$.

The class $A_{\nu}$ is significant because of the following theorem.

TheOREM 1.1. If $f(t)$ belongs to $A_{\nu}$, then the series

$$
x^{-\nu} s_{\nu}(x)=\sum_{n=0}^{\infty} 2(\nu+2 n+1) x^{-\nu} J_{\nu+2 n+1}(x) \int_{0}^{\rightarrow \infty} t^{-1} f(t) J_{\nu+2 n+1}(t) d t
$$

defines an entire function $x^{-\nu} s_{\nu}(x)$.

It is not difficult to extend the analysis in [9, pp. 533-535] to which we have already referred and to prove the following theorem.

Theorem 1.2. Let $\nu=0$ or -1 and $x>0$. If $f(t)$ belongs to $A_{\nu}$ and is $a b$ solutely continuous on $(0, x)$ then we have that

$$
s_{v}(x)=f(x-)-f(0+) J_{0}(x)-\int_{0}^{x} J_{0}(x-v) F(v) d v,
$$

where $F(v)$ is defined by equation (1.3) and $f(t)$ is defined for negative values of $t$ as $-(-1)^{v} f(-t)$.

An attempt to generalize this result to $A_{\nu}$ and $s_{\nu}$ fails, as we shall see 
in $\S 8$, because of the exceptional character of the values 0 and -1 for $\nu$ in an analytical expression for the particular Neumann series

$$
\sum_{n=0}^{\infty} 2(\nu+2 n+1) J_{\nu+2 n+1}(x) J_{\nu+2 n+1}(t) .
$$

Since this is the case other methods must be devised to handle the situation when $\nu(\nu+1) \neq 0$. These methods are found by generalizing a result of Bateman [1] who discovered an entirely different analytical expression for the series (1.8) when $\nu=0$. The result obtained in this manner is stated in the following theorem.

Theorem 1.3. Suppose that $x>0$ and that either $\nu>-1$ or $\nu$ is an integer. Then if $f(t)$ belongs to $\mathcal{A}_{\nu}$ and is of bounded variation in some neighborhood of $x$, we have that

$$
\begin{aligned}
s_{\nu}(x)= & \frac{1}{2}\{f(x+)+f(x-)\} \\
& -x \lim _{N=\infty, a=0} \int_{1}^{\rightarrow \infty} r J_{\nu}(x r) d r \int_{a}^{N} f(t) J_{\nu}(t r) d t .
\end{aligned}
$$

The proofs of these three theorems depend upon a number of auxiliary results on Bessel functions which are stated as lemmas and proved in $\$ \S 2,3,4$ and 5. Although $A_{\nu}$ is defined only for real values of $\nu$, we have endeavored to establish some of these auxiliary results for as wide a class of values of $\nu$ as possible. In particular this is true in $\$ 2$ where we shall derive various analytical expressions for the sum of the series (1.8). The proof of Theorem 1.1 is given in $\S 6$ and the proof of Theorem 1.2 in $\$ 7$. A generalization of the methods used to prove Theorem 1.2 is carried as far as possible when $\nu(\nu+1) \neq 0$ in $\S 8$. In the proof of Theorem 1.3 we need to know conditions on $\nu$ and $g(t)$ which insure the validity of the Hankel formula [9, p. 456]

$$
g(x)=\int_{0}^{\infty} r J_{\nu}(x r) d r \int_{0}^{\infty} t g(t) J_{\nu}(t r) d t,
$$

when $g(t)$ is zero except on an interval interior to $(0, \infty)$. A result of this nature which is somewhat more general than that stated by Watson [9, p. 456] is stated and proved in $\$ 9$. With the help of this result Theorem 1.3 is proved quite easily in $\$ 10$. If $f(t)$ is subjected to more stringent integrability requirements, the limit in equation (1.9) may be written as $\int_{1}^{\infty} r J_{\nu}(x r) d r \int_{0}^{\infty} f(t) J_{\nu}(t r) d t$. A result of this nature is given in $\$ 11$, and the methods used to prove it are also used to prove that the Hankel formula (1.10) holds under considerably weaker hypotheses than those generally assumed. In $\$ 12$ we consider very briefly expansions of functions $f(x)$ defined over $(-\infty, \infty)$ into series of the form 


$$
\sum_{n=0}^{\infty} c_{n} J_{n}(x)
$$

Finally, in $\S 13$ we discuss two simple examples when $\nu=0$.

2. Summation of a particular Neumann series. Of fundamental importance for our theory is the series

$$
\phi_{\nu}(x, t)=\sum_{n=0}^{\infty} 2(\nu+2 n+1) J_{\nu+2 n+1}(x) J_{\nu+2 n+1}(t) .
$$

This series is the sum of the alternate terms of the series

$$
\psi_{\nu}(x, t)=\sum_{n=0}^{\infty} 2(\nu+n) J_{\nu+n}(x) J_{\nu+n}(t)
$$

Kapteyn [3] deduced the formula for $\phi_{0}(x, t)$ which is given in Lemma 2.1 below and Watson [9, pp. 532-533] used similar methods to derive equation (2.3) for $\psi_{\nu}(x, t)$, although this formula is not so generally valid as he asserted. Titchmarsh [7, pp. 329-331] has used entirely different methods to obtain equation (2.4) for $\psi_{0}(x, t)$. A third approach was made by Bateman [1] who obtained (with a typographical error) $(2.10)$ for $\phi_{0}(x, t)$ given in Lemma 2.3.

We are going to prove the following lemma.

LEMмA 2.1. If $\nu$ is an integer such that $\nu(\nu+1) \neq 0$, then

$$
\begin{aligned}
\phi_{\nu}(x, t)= & \frac{1}{2} x \nu \int_{0}^{t} J_{\nu+1}(t-v)\left\{(x-v)^{-1} J_{\nu}(x-v)\right. \\
& \left.-(x+v)^{-1} J_{\nu}(x+v)\right\} d v \\
& +\frac{1}{2} x(\nu+1) \int_{0}^{t} J_{\nu}(t-v)\left\{(x-v)^{-1} J_{\nu+1}(x-v)\right. \\
& \left.+(x+v)^{-1} J_{\nu+1}(x+v)\right\} d v .
\end{aligned}
$$

This formula is still valid when $-1<R(\nu)$ provided that the real parts of $x, t$ and $x-t$ are all positive. In the exceptional cases when $\nu(\nu+1)=0$ we have that

$$
\begin{aligned}
\phi_{0}(x, t)= & \frac{1}{2} x \int_{0}^{t} J_{0}(t-v)\left\{(x-v)^{-1} J_{1}(x-v)\right. \\
& \left.+(x+v)^{-1} J_{1}(x+v)\right\} d v, \\
\phi_{-1}(x, t)= & \frac{1}{2} x \int_{0}^{t} J_{0}(t-v)\left\{(x-v)^{-1} J_{1}(x-v)\right. \\
& \left.-(x+v)^{-1} J_{1}(x+v)\right\} d v .
\end{aligned}
$$

Lemma 2.1 is an immediate consequence of the following lemma.

LEMMA 2.2. If $\nu$ is an integer such that $\nu(\nu-1) \neq 0$, then 


$$
\begin{aligned}
\psi_{\nu}(x, t)= & x(\nu-1) \int_{0}^{t}(x-v)^{-1} J_{\nu-1}(x-v) J_{\nu}(t-v) d v \\
& +x \nu \int_{0}^{t}(x-v)^{-1} J_{\nu}(x-v) J_{\nu-1}(t-v) d v
\end{aligned}
$$

This formula is still valid when $0<R(\nu)$ provided that the real parts of $x, t$ and $x-t$ are all positive. In the exceptional cases when $\nu(\nu-1)=0$ we have that

$$
\psi_{0}(x, t)=\psi_{1}(x, t)=x \int_{0}^{t}(x-v)^{-1} J_{1}(x-v) J_{0}(t-v) d v .
$$

To deduce Lemma 2.1 from Lemma 2.2 we merely observe that $\phi_{\nu}(x, t)$ $=2^{-1}\left[\psi_{\nu+1}(x, t)+e^{-\pi i(\nu+1)} \psi_{\nu+1}(-x, t)\right]$.

Watson $[9$, p. 533] asserted that equation (2.3) was valid provided that $R(\nu)>0$. But it is quite clear that the right-hand side of this equation fails to exist when $0<R(\nu)<1$ and $x<t$ because of the singularity of the integrand at $v=x$. Moreover, if $\nu=1 / 2$ and $x$ and $t$ are equal real numbers, the righthand side of equation (2.3) vanishes identically, whereas the left-hand side is surely positive. In view of these remarks it seems worthwhile to examine carefully the method of proof Watson suggests to find out just what is true.

The main tool is the formula $[9, \mathrm{p} .176]$

$$
J_{\nu}(z)=(1 / 2 \pi i) \int_{C_{\nu}} u^{-\nu-1} \exp \left\{\frac{1}{2} z\left(u-u^{-1}\right)\right\} d u,
$$

where $C_{\nu}$ is the familiar loop contour beginning at $-\infty$ in the third quadrant, circling the origin once in the positive direction, and returning to $-\infty$ in the second quadrant. This formula is valid for an arbitrary $\nu$ when $R(z)>0$. When $\nu$ is an integer we can, and do, choose $C_{\nu}$ as a circle with center at the origin $[9$, p. 20] and then the formula is valid for all values of $z$.

When $R(x)>0$ and $R(t)>0$ we have from the identity $[9, \mathrm{p} .45]$

$$
\begin{aligned}
\psi_{\nu}(x, t)= & \frac{1}{2} x \sum_{n=0}^{\infty}\left\{J_{\nu+n+1}(x)+J_{\nu+n-1}(x)\right\} J_{\nu+n}(t) \\
= & \frac{-x}{8 \pi^{2}} \int_{C_{\nu}} \int_{C_{\nu}} \sum_{n=0}^{\infty}\left(s^{-\nu-n-2}+s^{-\nu-n}\right) u^{-\nu-n-1} \\
& \quad \times \exp \left\{\frac{1}{2} x\left(s-s^{-1}\right)+\frac{1}{2} t\left(u-u^{-1}\right)\right\} d u d s \\
= & \left.\frac{x}{8 \pi^{2}} \int_{C_{\nu}} \int_{C_{\nu}} \frac{\left(s^{2}+1\right) \exp \left\{x\left(s-s^{-1}\right) / 2+t\left(u-u^{-1}\right) / 2\right.}{s^{\nu+1} u^{\nu}(s u-1)}\right\} d u d s,
\end{aligned}
$$


the interchange of summation and integration being valid provided that $|s u| \geqq A>1$, where $s$ and $u$ are any points on their respective contours. Let us define

$$
I(t)=\frac{1}{2 \pi i} \int_{C_{\nu}} u^{-\nu}(s u-1)^{-1} \exp \left\{\frac{1}{2} t\left(u-u^{-1}\right)\right\} d u .
$$

Then we find that

$$
\begin{aligned}
I^{\prime}(t)+\frac{1}{2}\left(s-s^{-1}\right) I(t) & =\frac{1}{4 \pi i} \int_{C_{\nu}}\left(s^{-1}+u^{-1}\right) u^{-\nu} \exp \left\{\frac{1}{2} t\left(u-u^{-1}\right)\right\} d u \\
& =\frac{1}{2} s^{-1} J_{\nu-1}(t)+\frac{1}{2} J_{\nu}(t), \\
I(0) & =\frac{1}{2 \pi i} \int_{C_{\nu}} u^{-\nu}(s u-1)^{-1} d u .
\end{aligned}
$$

We shall show presently that $I(0)=0$ when $R(\nu)>0$, although it is clear that $I(0)=s^{\nu-1}$ when $\nu$ is a nonpositive integer (for in this case $1 / s$ lies inside the circle $C_{\nu}$ and is the only singularity of the integrand). Taking this result for granted we conclude that when $R(\nu)>0$

$$
\begin{aligned}
\psi_{\nu}(x, t)= & \frac{x}{8 \pi i} \int_{C_{\nu}} \int_{0}^{t} s^{-1-\nu}\left(s^{2}+1\right) \exp \left\{\frac{1}{2}\left(s-s^{-1}\right)(x-v)\right\} \\
& \times\left\{J_{\nu}(t-v)+s^{-1} J_{\nu-1}(t-v)\right\} d v d s .
\end{aligned}
$$

If $R(\nu)>0$ and $R(x-t)>0$ we may interchange the order of integration in equation (2.7) and find with the help of equation (2.5) that

$$
\begin{aligned}
\psi_{\nu}(x, t)= & \frac{1}{4} x \int_{0}^{t} J_{\nu}(t-v)\left[J_{\nu-2}(x-v)+J_{\nu}(x-v)\right] d v \\
& +\frac{1}{4} x \int_{0}^{t} J_{\nu-1}(t-v)\left[J_{\nu-1}(x-v)+J_{\nu+1}(x-v)\right] d v .
\end{aligned}
$$

In view of equation (2.6), equations (2.8) and (2.3) are equivalent.

This verifies equation (2.3) when the real parts of $\nu, x, t$ and $x-t$ are all positive. When $\nu$ is a positive integer, equation (2.7) is valid for all values of $x$ and $t$. When $\nu$ is a nonpositive integer, an extra term of the form

$$
\frac{x}{4 \pi i} \int_{C_{\nu}} I(0)\left(s^{2}+1\right) v^{-\nu-1} \exp \left\{\frac{1}{2}\left(s-s^{-1}\right)(x-t)\right\} d s
$$

must be added to the right-hand side of equation (2.7). Since $I(0)=s^{\nu-1}$, it is seen with the help of equations (2.5) and (2.6) that this term has the value zero. Hence equation (2.7) is valid for all values of $x$ and $t$ when $\nu$ is any 
integer. The interchange of order of integration is trivial since $C_{\nu}$ is a circle and so equation (2.8) is valid for all values of $x$ and $t$. When $\nu=0$ or 1 , equation (2.8) reduces to equation (2.4) and otherwise to equation (2.3). This completes the proof of Lemma 2.2 except for proving that $I(0)=0$ when $R(\nu)>0$.

To establish this result we use the well known result $[11$, p. 245$]$

$$
1 / \Gamma(\nu+m+1)=\frac{1}{2 \pi i} \int_{C_{\nu}} w^{-\nu-m-1} e^{w} d w .
$$

Set $w=\alpha u$ where $\alpha>0$. Then

$$
\begin{aligned}
\alpha^{\nu+m} / \Gamma(\nu+m+1) & =\frac{1}{2 \pi i} \int_{C_{\nu}} u^{-\nu-m-1} e^{\alpha u} d u \\
\alpha^{\nu} \sum_{m=0}^{\infty}(\alpha / s)^{m} / \Gamma(\nu+m+1) & =\frac{s}{2 \pi i} \int_{C_{\nu}} u^{-\nu}(s u-1)^{-1} e^{\alpha u} d u
\end{aligned}
$$

the interchange of integration and summation being valid since $|s u| \geqq A>1$. Since the integral on the right converges uniformly in $\alpha$ when $0 \leqq \alpha$ and $0<R(\nu)$, it approaches $I(0)$ as $\alpha$ approaches 0 through positive values. When $R(\nu)>0$ the left-hand side clearly approaches 0 . Therefore, $I(0)=0$ when $R(\nu)>0$, as desired.

We shall now generalize Bateman's analysis [1] and prove the fo!lowing lemma.

Lemma 2.3. For all values of $\nu, x$ and $t$ we have that

$$
\phi_{\nu}(x, t)=\left\{x t /\left(x^{2}-t^{2}\right)\right\}\left\{x J_{\nu+1}(x) J_{\nu}(t)-t J_{\nu}(x) J_{\nu+1}(t)\right\},
$$

so that when $R(\nu)>-1$ or $\nu$ is an integer we have that

$$
\phi_{\nu}(x, t)=x t \int_{0}^{1} J_{\nu}(x r) J_{\nu}(t r) r d r .
$$

We shall refer to the class of numbers $\nu$ which are either integers or have a real part greater than -1 so of ten that it is worthwhile to introduce the letter $V$ to stand for this class.

The proof we are about to give of Lemma 2.3 is not a generalization of Bateman's analysis when $\nu=0$. He used properties of Legendre polynomials which do not seem to have an obvious extension to the case when $\nu \neq 0$. Our proof depends upon the Lommel polynomials $R_{m \mu}(x)$ which are defined as $[9$, p. 296]

$$
R_{m \mu}(x)=\sum_{k=0}^{[m / 2]}(-1)^{k}\left(\frac{1}{2} x\right)^{2 k-m} \Gamma(\mu+m-k)(m-k) ! / k ! \Gamma(\mu+k)(m-2 k) !,
$$

where $[m / 2]$ is the largest integer which does not exceed $m / 2$. These poly- 
nomials have the property that $[9$, p. 294]

$$
J_{\nu+2 n+1}(x)=R_{2 n, v+1}(x) J_{\nu+1}(x)-R_{2 n-1, v+2}(x) J_{\nu}(x) .
$$

The equivalence of equations (2.9) and (2.10) when $\nu$ is in $V$ follows from $[9$, p. 134].

Let $\theta_{\nu}(x, t)$ denote the right-hand side of equation (2.9). Since $t^{-\nu} \theta_{\nu}(x, t)$ is an entire odd function of $t$ when $x \neq 0$, it follows from the theory outlined in the second paragraph of the introduction that $\theta_{\nu}(x, t)$ may be expanded into a convergent series of the form $\theta_{\nu}(x, t)=\sum_{n=0}^{\infty} A_{n v}(x) J_{v+2 n+1}(t)$. Moreover, if the power series for $t^{-\nu} \theta_{\nu}(x, t)$ is of the form

$$
t^{-\nu} \theta_{\nu}(x, t)=\sum_{m=0}^{\infty} B_{m \nu}(x) t^{2 m+1},
$$

then we have that

$$
A_{n \nu}(x)=2(\nu+2 n+1) \sum_{m=0}^{n} 2^{\nu+2 m} \Gamma(\nu+n+m+1) B_{m \nu}(x) /(n-m) !
$$

It is easy to show with the help of the power series for $J_{\nu}(t)$ and $J_{\nu+1}(t)$ that $[9$, p. 40]

$$
\begin{aligned}
B_{m \nu}(x)= & J_{\nu+1}(x) \sum_{j=0}^{m}(-1)^{i} x^{2 j-2 m} / 2^{\nu+2 i} j ! \Gamma(\nu+j+1) \\
& -J_{\nu}(x) \sum_{j=0}^{m-1}(-1)^{i} x^{2 j-2 m+1} / 2^{\nu+2 j+1} j ! \Gamma(\nu+j+2),
\end{aligned}
$$

whence it follows that

$$
A_{n \nu}(x)=2(\nu+2 n+1)\left\{F_{n \nu}(x) J_{\nu+1}(x)-G_{n \nu}(x) J_{\nu}(x)\right\},
$$

where $F_{n \nu}(x)$ and $G_{n \nu}(x)$ are defined as follows:

$$
\begin{aligned}
& F_{n \nu}(x)=\sum_{m=0}^{n} \sum_{j=0}^{m}(-1)^{j} \Gamma(\nu+n+m+1)\left(\frac{1}{2} x\right)^{2 j-2 m} / j ! \Gamma(\nu+j+1)(n-m) ! \\
& G_{n \nu}(x)=\sum_{m=0}^{n} \sum_{j=0}^{m-1}(-1)^{j} \Gamma(\nu+n+m+1)\left(\frac{1}{2} x\right)^{2 j-2 m+1} / j ! \Gamma(\nu+j+2)(n-m) !
\end{aligned}
$$

If $m=j+n-k$, we find that $F_{n \nu}(x)$ may be written as follows:

$$
\begin{aligned}
& \sum_{k=0}^{n}(\left(\frac{1}{2} x\right)^{2 k-2 n} \sum_{j=0}^{k}(-1)^{j} \Gamma(\nu+2 n+j-k+1) / j ! \Gamma(\nu+j+1)(k-j) ! \\
& \quad=\sum_{k=0}^{n}\left(\frac{1}{2} x\right)^{2 k-2 n} \Gamma(\nu+2 n-k+1) F(-k, \nu+2 n-k+1 ; \nu+1 ; 1) / k ! \Gamma(\nu+1)
\end{aligned}
$$




$$
\begin{aligned}
& =\sum_{k=0}^{n}\left(\frac{1}{2} x\right)^{2 k-2 n}(-1)^{k} \Gamma(\nu+2 n-k+1)(2 n-k) ! / \Gamma(\nu+1+k) k !(2 n-2 k) ! \\
& =R_{2 n, \nu+1}(x)
\end{aligned}
$$

Similarly, we prove that $G_{n \nu}(x)=R_{2 n-1, \nu+2}(x)$. It then follows from equations (2.11) and (2.12) that $A_{n \nu}(x)=2(\nu+2 n+1) J_{\nu+2 n+1}(x)$. This completes the proof of Lemma 2.3 .

3. Recursion formulas for the remainder in the asymptotic expansion of $J_{\mu}(t)$. We shall use quite frequently the expansion

$$
\begin{aligned}
J_{\mu}(t)= & (2 / \pi t)^{1 / 2}\left[\cos \left(t-\delta_{\mu}\right) \sum_{m=0}^{m_{p}}(-1)^{m}(\mu, 2 m) /(2 t)^{2 m}\right. \\
& \left.-\sin \left(t-\delta_{\mu}\right) \sum_{m=0}^{m_{p-1}}(-1)^{m}(\mu, 2 m+1) /(2 t)^{2 m+1}\right]+R_{\mu}(t ; p),
\end{aligned}
$$

where $\delta_{\mu}=\mu \pi / 2+\pi / 4, m_{p}=[(p-2) / 2]$ is the greatest integer not exceeding $(p-2) / 2$, and

$$
(\mu, q)=\Gamma(\mu+q+1 / 2) / q ! \Gamma(\mu-q+1 / 2) .
$$

Here $\mu$ is an arbitrary complex number. It is therefore important to investigate the behavior of the remainder $R_{\mu}(t ; p)$ as a function of $\mu$. Our first result is stated in the following lemma.

LEMMA 3.1. If $p$ is even we have that

$$
\begin{aligned}
R_{\mu+1}+R_{\mu-1}-2 \mu t^{-1} & R_{\mu} \\
& =2 \mu \alpha_{p} t^{1 / 2-p} \cos \left(t-\delta_{\mu}\right) \Gamma(\mu+p-1.5) / \Gamma(\mu-p+2.5),
\end{aligned}
$$

while if $p$ is odd we replace $\cos \left(t-\delta_{\mu}\right)$ by- $\sin \left(t-\delta_{\mu}\right)$. Here $\alpha_{p}$ is defined as follows: $\alpha_{p}=(-1)^{m_{p}}(2 / \pi)^{1 / 2} 2^{p-2}(p-2)$ !.

If we use equations (3.1) and (2.6) we find that the left-hand side of the above equation has the value

$$
\begin{aligned}
(2 / \pi t)^{1 / 2} \cos \left(t-\delta_{\mu}\right) & {\left[4 \mu A_{p}(-1)^{m_{p}}\left(\mu, 2 m_{p}\right) /(2 t)^{1+2 m_{p}}\right.} \\
& \left.-\sum_{m=0}^{m_{p-1}}(-1)^{m}\{(\mu+1,2 m+1)-(\mu-1,2 m+1)-4 \mu(\mu, 2 m)\} /(2 t)^{2 m+1}\right] \\
& -(2 / \pi t)^{1 / 2} \sin \left(t-\delta_{\mu}\right)\left[4 \mu\left(1-A_{p}\right)(-1)^{m_{p-1}}\left(\mu, 2 m_{p}\right) /(2 t)^{2+2 m_{p-1}}\right. \\
& \left.+\sum_{m=0}^{m_{p}}(-1)^{m}\{(\mu+1,2 m)-(\mu-1,2 m)-4 \mu(\mu, 2 m-1)\} /(2 t)^{2 m}\right]
\end{aligned}
$$


where $A_{p}=0$ or 1 according as $m_{p-1}=m_{p}$ or $m_{p-1}<m_{p}$, that is, according as $p$ is odd or even. It is easy to see that

$$
(\mu+1, q)-(\mu-1 ; q)-4 \mu(\mu, q-1)=0
$$

for every nonnegative integer $q$. The expression (3.3) therefore reduces to the right-hand side of the equation in the lemma.

The result of Lemma 3.1 clearly provides a generalization of equation (2.6) to which it reduces when $p=1$. A generalization of the identity $[9$, p. 45]

$$
2 J_{\mu}^{\prime}(x)-J_{\mu-1}(x)+J_{\mu+1}(x)=0
$$

is supplied by the following lemma.

LEMMA 3:2. If $p$ is even we have that

$$
\begin{aligned}
2 R_{\mu}^{\prime}-R_{\mu-1} & +R_{\mu+1} \\
& =(2 p-3) \alpha_{p} t^{1 / 2-p} \cos \left(t-\delta_{\mu}\right) \Gamma(\mu+p-1.5) / \Gamma(\mu-p+2.5),
\end{aligned}
$$

while if $p$ is odd we replace $\cos \left(t-\delta_{\mu}\right)$ by $-\sin \left(t-\delta_{\mu}\right)$.

If we use equation (3.4) we find that the left-hand side of the above equation multiplied by $(\pi t / 2)^{1 / 2}$ is equal to

$$
\begin{gathered}
\cos \left(t-\delta_{\mu}\right)\left[2\left(4 m_{p}+1\right) A_{p}(-1)^{m_{p}}\left(\mu, 2 m_{p}\right) /(2 t)^{1+2 m_{p}}\right. \\
+\sum_{m=0}^{m_{p-1}}(-1)^{m}\{2(\mu, 2 m+1)-(\mu-1,2 m+1)-(\mu+1,2 m+1) \\
\left.+(8 m+2)(\mu, 2 m)\} /(2 t)^{2 m+1}\right] \\
+\sin \left(t-\delta_{\mu}\right)\left[2\left(3+4 m_{p-1}\right)\left(A_{p}-1\right)(-1)^{m_{p}}\left(\mu, 1+2 m_{p-1}\right) /(2 t)^{2+2 m_{p-1}}\right] \\
+\sum_{m=0}^{m_{p}}(-1)^{m}\{2(\mu, 2 m)-(\mu-1,2 m)-(\mu+1,2 m) \\
\left.+(8 m-2)(\mu, 2 m-1)\} /(2 t)^{2 m}\right],
\end{gathered}
$$

where $A_{p}$ is defined as in the proof of Lemma 3.1. It is easy to see that

$$
2(\mu, q)-(\mu-1, q)-(\mu+1, q)+(4 q-2)(\mu, q-1)=0
$$

for every nonnegative integer $q$. The expression (3.5) therefore reduces to $(\pi t / 2)^{1 / 2}$ times the right-hand side of the equation in the lemma.

Although we shall use Lemma 3.1 in its entirety we shall only use Lemma 3.2 in order to prove the following lemma. 
LEMMA 3.3. As a function of $t$ we have that

$$
R_{\mu}(t ; p)=O\left(t^{1 / 2-p}\right), \quad R_{\mu}^{\prime}(t ; p)=O\left(t^{1 / 2-p}\right) .
$$

The first equation is well known $[9,198-199]$. The second equation follows from Lemma 3.2 and the first equation.

It is quite plain that by mathematical induction we can show that $R_{\mu}^{(q)}(t ; p)=O\left(t^{1 / 2-p}\right)$ for any nonnegative integer $q$.

4. Some auxiliary lemmas. In this section we shall prove a few miscellaneous results needed for the proofs of the main theorems.

LEMMA 4.1. There exist nonpositive functions $a_{q}(x, p)(0 \leqq q \leqq p-2 ; 0<x)$ such that if

$$
u_{\mu}(x, p)=\sum_{q=0}^{p-2} a_{q}(x, p) \mu^{2 q}
$$

then we have that

$$
\begin{aligned}
u_{\mu+1}-2 \mu x^{-1} u_{\mu}- & u_{\mu-1} \\
& \geqq 2 \mu\left|\alpha_{p} \Gamma(\mu+p-1.5) / \Gamma(\mu-p+2.5)\right| \quad(\mu \geqq 2) .
\end{aligned}
$$

Define $\beta_{p}$ as $\max _{\mu \geqq 2}\left|\Gamma(\mu+p-1.5) / \mu^{2 p-4} \Gamma(\mu-p+2.5)\right|$. The existence of $\beta_{p}$ follows from well known properties of the gamma function [8, p. 58]. We define $a_{q}(x, p)$ by recursion as follows:

$$
a_{p-2}(x, p)=-x\left|\alpha_{p}\right| \beta_{p}, \quad a_{q}(x, p)=x \sum_{m=q+1}^{p-2} a_{m}(x, p)_{2 m} C_{2 q+1} .
$$

Hence $a_{q}(x, p) \leqq 0$. If $u_{\mu}(x, p)$ is defined by equation (4.1), then the lefthand side of the inequality (4.2) has the value $2\left|\alpha_{p}\right| \beta_{p} \mu^{2 p-3}$. This is seen in the following manner:

$$
\begin{aligned}
\sum_{m=0}^{p-2} a_{m}(x, p)\left[(\mu+1)^{2 m}-(\mu-1)^{2 m}\right]-2 x^{-1} \sum_{q=0}^{p-2} a_{q}(x, p) \mu^{2 q+1} \\
=2 \sum_{m=0}^{p-2} a_{m}(x, p) \sum_{q=0}^{m-1}{ }_{2 m} C_{2 q+1} \mu^{2 q+1}-2 x^{-1} \sum_{q=0}^{p-2} a_{q}(x, p) \mu^{2 q+1} \\
=2 \sum_{q=0}^{p-3} \mu^{2 q+1} \sum_{m=q+1}^{p-2} a_{m}(x, p)_{2 m} C_{2 q+1}-2 x^{-1} \sum_{q=0}^{p-2} a_{q}(x, p) \mu^{2 q+1} \\
=-2 x^{-1} a_{p-2}(x, p) \mu^{2 p-3}=2\left|\alpha_{p}\right| \beta_{p} \mu^{2 p-3}
\end{aligned}
$$

by virtue of equation (4.3). The inequality (4.2) now follows from the definition of $\beta_{p}$.

LEMMA 4.2. There exists a nonnegative function $B_{p}(x)$ such that

$$
t^{p-1 / 2}\left|R_{\mu}(t ; p)\right| \leqq B_{p}(x) K_{\mu}(x)+u_{\mu}(x, p)
$$


whenever $t \geqq x>0$ and $\mu \geqq 0$.

Define $\lambda$ to be $\mu-[\mu]$, so that $0 \leqq \lambda<1$, and then define $B_{p}(x)$ so that

$$
B_{p}(x) K_{0}(x)=-u_{2}(x, p)+\underset{t \geqq x, 0 \leqq \mu \leqq 2}{\text { l.u.b. }}\left\{t^{p-1 / 2}\left|R_{\mu}(t ; p)\right|\right\} .
$$

It follows from this definition that $B_{p}(x) \geqq 0$ and, when $t \geqq x$, that

$$
\begin{aligned}
t^{p-1 / 2}\left|R_{\lambda}(t ; p)\right| & \leqq u_{2}(x, p)+B_{p}(x) K_{0}(x), \\
t^{p-1 / 2}\left|R_{\lambda+1}(t ; p)\right| & \leqq u_{2}(x, p)+B_{p}(x) K_{0}(x) .
\end{aligned}
$$

Since $a_{q}(x, p) \leqq 0, u_{\mu}(x, p)$ is a decreasing function of $\mu$. Therefore,

$$
u_{2}(x, p) \leqq u_{\lambda+1}(x, p) \leqq u_{\lambda}(x, p) \text {. }
$$

Moreover, it follows from the identity $[9$, p. 185]

$$
K_{\mu}(x)=\int_{0}^{\infty} \cosh \mu y \exp (-x \cosh y) d y
$$

that $K_{0}(x) \leqq K_{\lambda}(x) \leqq K_{\lambda+1}(x)$. We conclude that the inequality (4.4) holds when $\mu$ is replaced by $\lambda$ or by $\lambda+1$, and hence whenever $0 \leqq \mu<2$. Suppose that $\mu \geqq 2$ and that the inequality (4.4) holds for $\mu$ and for $\mu-1$. Then we have from Lemma 3.1 when $t \geqq x$ that

$$
\begin{aligned}
t^{p-1 / 2}\left|R_{\mu+1}(t ; p)\right| \leqq & 2 \mu x^{-1} t^{p-1 / 2}\left|R_{\mu}(t ; p)\right|+t^{p-1 / 2}\left|R_{\mu-1}(t ; p)\right| \\
& +2 \mu\left|\alpha_{p} \Gamma(\mu+p-1.5) / \Gamma(\mu-p+2.5)\right| \\
\leqq & B_{p}(x)\left\{2 \mu x^{-1} K_{\mu}(x)+K_{\mu-1}(x)\right\}+u_{\mu+1}(x, p) \\
& -\left[u_{\mu+1}(x, p)-2 \mu x^{-1} u_{\mu}(x, p)-u_{\mu-1}(x, p)\right. \\
& \left.-2 \mu\left|\alpha_{p} \Gamma(\mu+p-1.5) / \Gamma(\mu-p+2.5)\right|\right] .
\end{aligned}
$$

Since $\left[9\right.$, p. 79] the coefficient of $B_{p}(x)$ is $K_{\mu+1}(x)$, and since we have from Lemma 4.1 that the term inside the square brackets is nonnegative, it follows that the inequality (4.4) holds for $\mu+1$. This completes the induction and verifies Lemma 4.2 for all nonnegative values of $\mu$.

LEMma 4.3. When $x>0$ and $\mu>0$ we have that

$$
K_{\mu}(2 x) \leqq \frac{1}{2} \Gamma(\mu) x^{-\mu} .
$$

We use the identity $[9$, p. 185$]$

$$
K_{\mu}(x)=\Gamma\left(\mu+\frac{1}{2}\right)\left(\frac{1}{2} x\right)^{-\mu} \pi^{-1 / 2} \int_{0}^{\infty}\left(u^{2}+1\right)^{-\mu-1 / 2} \cos x u d u
$$

to conclude that 


$$
K_{\mu}(2 x) \leqq \Gamma\left(\mu+\frac{1}{2}\right) x^{-\mu} \pi^{-1 / 2} \int_{0}^{\infty}\left(u^{2}+1\right)^{-\mu-1 / 2} d u=\frac{1}{2} \Gamma(\mu) x^{-\mu} .
$$

LEMMA 4.4. When $x \geqq 0$ and $\mu \geqq 0$ we have that

$$
\left|J_{\mu}(x)\right| \leqq\left(\frac{1}{2} x\right)^{\mu} / \Gamma\left(\mu+\frac{1}{2}\right) .
$$

This result is well known $[9$, p. 49].

5. Some general remarks about Neumann series. Before we take up the proof of Theorem 1.1 in the next section, it is convenient to amplify some remarks of Pincherle [6] (see also [9, pp. 526-527]) on the region of convergence of arbitrary Neumann series of the form (1.1) in which $\nu$ is a real number and we do not assume the existence of a function $f(t)$ in terms of which $a_{n \nu}$ may be defined by equation (1.2). We first prove the following lemma.

Lемма 5.1. If the series (1.1) converges when $x=\beta$, then

$$
a_{n \nu}=o\left[\Gamma(\nu+2 n+2)(2 / \beta)^{\nu+2 n+1}\right] .
$$

Therefore, the series

$$
\sum_{n=0}^{\infty} a_{n \nu} x^{-\nu} J_{\nu+2 n+1}(x)
$$

converges absolutely-uniformly to an analytic function when $|x|<|\beta|$.

If equation (5.1) did not hold, there would be a positive number $\epsilon$ and an increasing sequence $\left\{n_{k}\right\}$ of integers such that

$$
\left|a_{n_{k}, \nu}\right|>\epsilon \Gamma\left(\nu+2 n_{k}+2\right)(2 / \beta)^{\nu+1+2 n_{k}} .
$$

Since the series (1.1) converges when $x=\beta$, we have that

$$
\Gamma\left(\nu+2 n_{k}+2\right)(2 / \beta)^{\nu+1+2 n_{k}} J_{\nu+1+2 n_{k}}(\beta)=o(1) .
$$

But we know that $[9$, p. 44$]$

$$
J_{\nu+1+2 n_{k}}(\beta)=\left\{\left(\frac{1}{2} \beta\right)^{\nu+1+2 n_{k}} / \Gamma\left(\nu+2 n_{k}+2\right)\right\}\left\{1+\theta_{k}\right\},
$$

in which (for sufficiently large $k$ )

$$
\left|\theta_{k}\right|<\exp \left\{\frac{1}{4}|\beta|^{2} /\left(\nu+2 n_{k}+2\right)\right\}-1 .
$$

Therefore, $\left|1+\theta_{k}\right|=o(1)$, so that $\lim _{k=\infty} \theta_{k}=-1$. Since it follows from the inequality (5.3) that $\theta_{k}$ approaches 0 and not -1 , we have reached a contradiction. This completes the proof that equation (5.1) is true. 
To prove the second part of the lemma, pick a number $M$ such that

$$
\left|a_{n \nu}\right| \leqq M \Gamma(\nu+2 n+2)(2 / \beta)^{\nu+2 n+1} \text {. }
$$

By virtue of Lemma 4.4 the series (5.2) is dominated by the series

$$
M|\beta|^{-\nu} \sum_{n=0}^{\infty}|x / \beta|^{2 n+1}<+\infty
$$

when $|x|<|\beta|$ and $\nu>-1$. If $\nu \leqq-1$ we apply the same argument to the infinite series obtained by omitting the first $1+[(-\nu-1) / 2]$ terms of the series (1.1). This completes the proof of the lemma.

LEMMA 5.2. If the series (1.1) converges to 0 on any interval of the real axis $\left({ }^{3}\right)$, the coefficients $a_{n v}$ must all vanish.

For in this case the series (5.2) defines an analytic function $\alpha(x)$ which vanishes everywhere in its region of analyticity, and in particular in a neighborhood of the origin. Since the series is uniformly convergent we may compute the derivatives of $\alpha(x)$ at the origin by termwise differentiation. Thus we find that

$$
\begin{aligned}
0 & =\alpha^{(2 p+1)}(0)=\left.\sum_{n=0}^{\infty} a_{n \nu}\left\{x^{-\nu} J_{\nu+2 n+1}(x)\right\}^{(2 p+1)}\right|_{x=0} \\
& =\sum_{n=0}^{p}(-1)^{p-n} a_{n \nu}\left(\frac{1}{2}\right)^{\nu+2 p+1}(2 p+1) ! /(p-n) ! \Gamma(\nu+n+p+2) .
\end{aligned}
$$

We now find by an easy induction that $a_{n \nu}=0(n=0,1, \cdots)$.

6. Proof of Theorem 1.1. By virtue of Lemma 5.1 the proof of Theorem 1.1 requires merely a demonstration that the series (1.1) converges for every positive value of $x$ when $\nu$ is real, $f(t)$ is in $A_{\nu}$, and

$$
a_{n \nu}=2(\nu+2 n+1) \int_{0}^{\rightarrow \infty} t^{-1} f(t) J_{\nu+2 n+1}(t) d t .
$$

Suppose first that $\nu>-1$. If we define

$$
\begin{aligned}
& a_{n \nu}^{\prime}(a)=2(\nu+2 n+1) \int_{0}^{a} t^{-1} f(t) J_{\nu+2 n+1}(t) d t, \\
& a_{n \nu}^{\prime \prime}(N)=2(\nu+2 n+1) \int_{N}^{\rightarrow \infty} t^{-1} f(t) J_{\nu+2 n+1}(t) d t,
\end{aligned}
$$

then $a_{n \nu}=a_{n \nu}^{\prime \prime}(2 x)+a_{n \nu}^{\prime}(2 x)$. We see from Lemma 4.4 that

(3) It is clear that this lemma may be extended to other sets of points besides real intervals. The present lemma, however, is sufficient for the discussion of the uniqueness of representation of a function on $(0, \infty)$ by a Neumann series. 


$$
\left|a_{n \nu}^{\prime}(2 x)\right| \leqq\left\{x^{2 n} / 2^{\nu} \Gamma(\nu+2 n+1)\right\} \int_{0}^{2 x} t^{\nu}|f(t)| d t .
$$

If we apply Lemma 4.4 again and remember that $f(t)$ is in $A_{\nu}$ we conclude that the series

$$
\sum_{n=0}^{\infty} a_{n v}^{\prime}(2 x) J_{\nu+2 n+1}(x)
$$

is convergent.

If we now use equation (3.1) with $\mu$ replaced by $\nu+2 n+1$ we find that

$$
\begin{aligned}
\left|a_{n \nu}^{\prime \prime}(2 x)\right| & \leqq 2(\nu+2 n+1)\left[\int_{2 x}^{\rightarrow \infty} t^{-1}\left|f(t) R_{\nu+2 n+1}(t ; p)\right| d t\right. \\
& +\left(\frac{2}{\pi}\right)^{1 / 2}\left\{\sum_{m=0}^{m_{p}} C_{\nu}(m, 2 x)(\nu+2 n+1,2 m) / 2^{2 m}\right. \\
& \left.\left.+\sum_{m=0}^{m_{p}-1} S_{\nu}(m, 2 x)(\nu+2 n+1,2 m+1) / 2^{2 m+1}\right\}\right],
\end{aligned}
$$

where $C_{\nu}(m, N)$ and $S_{\nu}(m, N)$ are defined as follows:

$$
\begin{aligned}
& C_{\nu}(m, N)=\left|\int_{N}^{\rightarrow \infty} t^{(4 m+3) / 2} f(t) \cos \left(t-\frac{1}{2} \nu \pi+\frac{1}{4} \pi\right) d t\right|, \\
& S_{\nu}(m, N)=\left|\int_{N}^{\rightarrow \infty} t^{-(4 m+5) / 2} f(t) \sin \left(t-\frac{1}{2} \nu \pi+\frac{1}{4} \pi\right) d t\right| .
\end{aligned}
$$

Since $f(t)$ is in $A_{\nu}$, there is a number $M_{\nu}(N)$ such that

$$
\begin{gathered}
C_{\nu}(m, N) \leqq M_{\nu}(N), \quad S_{\nu}(m, N) \leqq M_{\nu}(N) \quad\left(0 \leqq m \leqq m_{p}\right), \\
\int_{N}^{\infty} t^{-p-1 / 2}|f(t)| d t \leqq M_{\nu}(N) .
\end{gathered}
$$

It now follows from Lemmas 4.2 and 4.3 that

$$
\begin{aligned}
& \int_{2 x}^{\infty} t^{-1}\left|f(t) R_{v+2 n+1}(t ; p)\right| d t \\
& \leqq M_{\nu}(2 x)\left[B_{p}(2 x) K_{v+2 n+1}(2 x)+u_{v+2 n+1}(2 x, p)\right] \\
& \leqq M_{\nu}(2 x)\left[B_{p}(2 x) \Gamma(\nu+2 n+1) / 2 x^{v+2 n+1}+u_{v+2 n+1}(2 x, p)\right] .
\end{aligned}
$$

We conclude from the above inequalities and Lemma 4.4 that the series

$$
\sum_{n=0}^{\infty} a_{n v}^{\prime \prime}(2 x) J_{v+2 n+1}(x)
$$


is dominated by

$\begin{aligned} 2 M_{\nu}(2 x) \sum_{n=0}^{\infty}\left\{\left(\frac{1}{2} x\right)^{\nu+2 n+1}\right. & / \Gamma(\nu+2 n+1)\}\left\{B_{p}(2 x) \Gamma(\nu+2 n+1) / 2 x^{\nu+2 n+1}\right. \\ & +6.4) \\ & \left.+u_{\nu+2 n+1}(2 x ; p)+(2 / \pi)^{1 / 2} \sum_{k=0}^{p-2}(\nu+2 n+1, k) / 2^{k}\right\} .\end{aligned}$

If we examine the definitions (4.1) and (3.2) of $u_{\mu}(x, p)$ and $(\mu, q)$ we see that this last series is convergent.

It follows that the series (1.6) is convergent and defines an entire function when $\nu>-1$. The proof when $\nu \leqq-1$ may be made with the help of the identity,

(6.5) $s_{\nu}(x)=\sum_{n=0}^{q-1} 2(\nu+2 n+1) J_{\nu+2 n+1}(x) \int_{0}^{\rightarrow \infty} t^{-1} f(t) J_{\nu+2 n+1}(t) d t+s_{\nu+2 q}(x)$,

which is valid for any integer $q$ such that $q \geqq 1$. If $\nu \leqq-1$ we may define $q$ to be $1+[(-\nu-1) / 2]$. Then $\nu+2 q>-1$. Since $A_{\nu}$ is a subset of $A_{\nu+2 q}$ it follows from our previous analysis that $x^{-\nu-2 q} S_{\nu+2 q}(x)$, and hence also $x^{-v} s_{\nu+2 q}(x)$, is an entire function. Since the finite series in equation (6.5) obviously becomes an entire function after multiplication by $x^{-\nu}$, we conclude that $x^{-\nu} s_{\nu}(x)$ is an entire function for any real value of $\nu$. These remarks complete the proof of Theorem 1.1.

The analysis used to prove Theorem 1.1 may be modified slightly and used to prove the following lemma which asserts the possibility of interchanging the order of integration and summation in the definition of $s_{\nu}(x)$.

Lemma. 6.1. If $f(t)$ is in $A$, and $x>0$, then

$$
s_{\nu}(x)=\int_{0}^{\rightarrow \infty} t^{-1} f(t) \sum_{n=0}^{\infty} 2(\nu+2 n+1) J_{\nu+2 n+1}(x) J_{\nu+2 n+1}(t) d t .
$$

Since the series

$$
t^{-1-\nu} \phi_{\nu}(x, t)=\sum_{n=0}^{\infty} 2(\nu+2 n+1) J_{\nu+2 n+1}(x) J_{\nu+2 n+1}(t) t^{-1-\nu}
$$

converges uniformly in $t$ on any finite interval, and since $t^{\nu} f(t)$ is integrable over any finite interval, it is sufficient to show that

$$
\lim _{N=\infty} \sum_{n=0}^{\infty}\left|a_{n \nu}^{\prime \prime}(N) J_{\nu+2 n+1}(x)\right|=0,
$$

in which $a_{n \nu}^{\prime \prime}(N)$ is defined by equation (6.2). Since $f(t)$ is in $A_{\nu}$, for each positive $\epsilon$ we can choose $N_{0}$ so large that $M_{\nu}(N)<\epsilon$ and $N>2 x$ when $N>N_{0}$ 
and $\nu>-1$. We conclude that the series (6.7) is dominated by the series (6.4) in which the term $M_{\nu}(2 x)$ is replaced by $\epsilon$. This proves the relation (6.7) and hence also the lemma when $\nu>-1$. When $\nu \leqq-1$ we merely apply the idea behind equation (6.5) to see that the relation (6.7) still holds.

For future reference we note as a consequence of the inequality (6.3) that when $f(t)$ is in $\mathcal{A}$, we have that

$$
\lim _{a=\infty} \sum_{n=0}^{\infty}\left|a_{n \nu}^{\prime}(a) J_{v+2 n+1}(x)\right|=0,
$$

in which $a_{n \nu}^{\prime}(a)$ is defined by equation (6.1).

7. Proof of Theorem 1.2. From Lemmas 6.1 and 2.1 we conclude when $\nu=0$ or -1 that

$$
s_{\nu}(x)=\frac{1}{2} \int_{0}^{\rightarrow \infty} f(t) d t \int_{0}^{x} L_{\nu}(t, v) J_{0}(x-v) d v,
$$

where $L_{\nu}(t, v)$ is defined as follows:

$$
L_{\nu}(t, v)=(t-v)^{-1} J_{1}(t-v)+(-1)^{\nu}(t+v)^{-1} J_{1}(t+v) .
$$

If it is permissible to interchange the order of integration we find that

$$
\begin{aligned}
s_{v}(x)= & \frac{1}{2} \int_{0}^{x} J_{0}(x-v) d v \int_{0}^{\rightarrow \infty} t^{-1} J_{1}(t)\{f(v+t)-f(v-t)\} d t \\
& +\int_{0}^{x} J_{0}(x-v) d v \int_{0}^{v} t^{-1} J_{1}(t) f(v-t) d t,
\end{aligned}
$$

provided that $f(t)$ be defined for negative values of $t$ as $-(-1)^{\prime} f(-t)$. This interchange will be valid provided that the integral

$$
\int_{0}^{\rightarrow \infty} L_{\nu}(t, v) f(t) d t
$$

converges uniformly in $v$ on the interval $(0, x)$. That this is so follows from the definition of the class $\mathcal{A}_{\nu}$ if we use equation (3.1) to see that

$$
\begin{aligned}
(\pi / 2)^{1 / 2} L_{\nu}(t, v)= & (\pi / 2)^{1 / 2}\left\{(t-v)^{-1} R_{1}(t-v ; p)\right. \\
& \left.+(-1)^{\nu}(t+v)^{-1} R_{1}(t+v ; p)\right\} \\
& -\left(A_{\nu} \cos v+B_{\nu} \sin v\right) \cos (t+\pi / 4) \\
& -\left(A_{v+1} \cos v-B_{v+1} \sin v\right) \sin (t+\pi / 4),
\end{aligned}
$$

in which $A$, and $B_{v}$ are defined as follows:

$$
A_{\nu}=\sum_{m=0}^{m_{p}}(-1)^{m}(1,2 m)\left\{(t-v)^{-(4 m+3) / 2}+(-1)^{\nu}(t+v)^{-(4 m+3) / 2}\right\} 2^{-(4 m+3) / 2}
$$




$$
\begin{aligned}
B_{\nu}=\sum_{m=0}^{m_{p-1}}(-1)^{m}(1,2 m+1)\left\{(t-v)^{-(4 m+5) / 2}\right. & \\
& \left.-(-1)^{\nu}(t+v)^{-(4 m+5) / 2}\right\} 2^{-(4 m+5) / 2} .
\end{aligned}
$$

We now transform the second integral in equation (7.1) with the help of the identity $[9$, p. 380 $] \int_{0}^{u} t^{-1} J_{1}(t) J_{0}(u-t) d t=J_{1}(u)$, and find that it is equal to

$$
\begin{aligned}
\int_{0}^{x} \int_{0}^{u} J_{0}(u-t) f(x-u) t^{-1} J_{1}(t) d t d v & =\int_{0}^{x} J_{1}(u) f(x-u) d u \\
= & f(x-)-f(0+) J_{0}(x)-\int_{0}^{x} J_{0}(u) f^{\prime}(x-u) d u \\
= & f(x-)-f(0+) J_{0}(x)-\int_{0}^{x} J_{0}(x-v) f^{\prime}(v) d v .
\end{aligned}
$$

Introducing the function $F(v)$ defined by equation (1.3) we see that equation (1.7) holds, as desired.

Corollary. Suppose that $\nu(\nu+1)=0$, that $f(t)$ belongs to $\mathcal{A}_{\nu}$, and that $f(t)$ is absolutely continuous on $(0, X)$. Then $f(x)=s_{\nu}(x)$ on the open interval $(0, X)$ if and only if

$$
f(0+)=0, \quad F(v) \equiv 0 \quad \text { on } \quad(0, X) .
$$

By virtue of equation (1.7) and the continuity of $f(x)$ when $0<x<X$ it is clear that $s_{\nu}(x)=f(x)$ when equations (7.2) hold. Suppose conversely that $s_{v}(x)=f(x)$ on $(0, X)$. Then on $(0, \mathrm{X})$

$$
f(0+) J_{0}(x)+\int_{0}^{x} J_{0}(x-v) F(v) d v=0 .
$$

Letting $x$ approach zero we find immediately that $f(0+)=0$, and hence that

$$
\int_{0}^{x} J_{0}(x-v) F(v) d v=0
$$

By Theorem 1.1, $F(v)$ is continuous on $(0, X)$ and so we may differentiate equation (7.3) to obtain

$$
F(x)=\int_{0}^{x} J_{1}(x-v) F(v) d v
$$

This equation is an integral equation of Volterra type of the second kind and hence $[5$, p. 14] has the unique continuous solution, $F(v) \equiv 0$ on $(0, X)$, since the kernel $J_{1}(x-v)$ is bounded.

8. Extension of Theorem 1.2 for a general values of $\nu$. When $\nu(\nu+1) \neq 0$ 
an attempt to obtain a result like that of Theorem 1.2 with the help of equation (2.1) evidently fails, since it is necessary to know $\phi_{\nu}(x, t)$ when $x-t<0$, and equation (2.1) does not hold when $x-t<0$ unless $\nu$ is an integer. Even then the best we can do is to prove the following theorem.

THEOREM 8.1. When $\nu$ is an integer such that $\nu(\nu+1) \neq 0$ and $f(t)$ is in $\mathcal{A}_{\nu}$, we have that

$$
\begin{aligned}
s_{\nu}(x)= & \frac{1}{2} \nu \int_{0}^{x} J_{\nu+1}(x-v) d v \int_{0}^{\rightarrow \infty} u^{-1} J_{\nu}(u)[f(u+v)-f(u-v)] d u \\
& +\frac{1}{2}(\nu+1) \int_{0}^{x} J_{\nu}(x-v) d v \int_{0}^{\rightarrow \infty} u^{-1} J_{\nu+1}(u)[f(u+v)+f(u-v)] d u
\end{aligned}
$$

provided that $f(t)$ be defined for negative values of $t$ as $-(-1)^{\prime} f(-t)$.

We conclude from Lemmas 6.1 and 2.1 that

$$
\begin{aligned}
s_{\nu}(x)= & \frac{1}{2} \nu \int_{0}^{\rightarrow \infty} f(t) d t \int_{0}^{x} H_{\nu}(t, v) J_{\nu+1}(x-v) d v \\
& +\frac{1}{2}(\nu+1) \int_{0}^{\rightarrow \infty} f(t) d t \int_{0}^{x} K_{\nu}(t, v) J_{\nu}(x-v) d v=\sigma_{\nu}^{\prime}+\sigma_{\nu}^{\prime},
\end{aligned}
$$

where $H_{\nu}(t, v)$ and $K_{\nu}(t, v)$ are defined as follows:

$$
\begin{aligned}
& H_{\nu}(t, v)=(t-v)^{-1} J_{\nu}(t-v)-(t+v)^{-1} J_{\nu}(t+v), \\
& K_{\nu}(t, v)=(t-v)^{-1} J_{\nu+1}(t-v)+(t+v)^{-1} J_{\nu+1}(t+v) .
\end{aligned}
$$

Manipulations like those in the preceding section show that

$$
\begin{aligned}
\sigma_{\nu}^{\prime}= & \frac{1}{2} \nu \int_{0}^{x} J_{\nu+1}(x-v) d v \int_{0}^{\rightarrow \infty} H_{\nu}(t, v) f(t) d t \\
= & \frac{1}{2} \nu \int_{0}^{x} J_{\nu+1}(x-v) d v \int_{0}^{\rightarrow \infty} u^{-1} J_{\nu}(u)[f(u+v)-f(u-v)] d u \\
& +\nu \int_{0}^{x} J_{\nu+1}(x-v) d v \int_{0}^{v} t^{-1} J_{\nu}(t) f(t-v) d t, \\
\sigma_{\nu}^{\prime \prime}= & \frac{1}{2}(\nu+1) \int_{0}^{x} J_{\nu}(x-v) d v \int_{0}^{\rightarrow \infty} K_{\nu}(t, v) f(t) d t \\
= & \frac{1}{2}(\nu+1) \int_{0}^{x} J_{\nu}(x-v) d v \int_{0}^{\rightarrow \infty} u^{-1} J_{\nu+1}(u)[f(u+v)+f(u-v)] d u \\
& -(\nu+1) \int_{0}^{x} J_{\nu}(x-v) d v \int_{0}^{v} t^{-1} J_{\nu+1}(t) f(t-v) d t,
\end{aligned}
$$


the inversions of order of integration being justified by the uniform convergence of the integrals

$$
\int_{0}^{\rightarrow \infty} H_{\nu}(t, v) f(t) d t, \quad \int_{0}^{\rightarrow \infty} K_{\nu}(t, v) f(t) d t
$$

when $0 \leqq v \leqq x$. Since we have when $\nu$ is a nonzero integer and $\mu>-1$ that $[9$, p. 380]

$$
\nu \int_{0}^{u} t^{-1} J_{\mu}(u-t) J_{\nu}(t) d t=J_{\mu+\nu}(u)
$$

it is clear when $\nu(\nu+1) \neq 0$ that

$$
\begin{aligned}
\nu \int_{0}^{x} J_{\nu+1}(x-v) d v \int_{0}^{v} t^{-1} J_{\nu}(t) & f(t-v) d t \\
& =(\nu+1) \int_{0}^{x} J_{\nu}(x-v) d v \int_{0}^{v} t^{-1} J_{\nu+1}(t) f(t-v) d t .
\end{aligned}
$$

Theorem 8.1 now follows from the definitions of $\sigma_{\nu}^{\prime}$ and $\sigma_{\nu}^{\prime \prime}$.

9. An elementary Hankel formula. In order to prove Theorem 1.3 we shall need the Hankel identity

$$
g(x)=\int_{0}^{\infty} r J_{\nu}(x r) d r \int_{0}^{\infty} t g(t) J_{\nu}(t r) d t
$$

which is known $[9$, p. 456], $[7$, p. 240] to hold under certain restrictions on $\nu$ and $g(t)$. Ultimately (see Theorem 11.2 below) we are going to establish the validity of equation (9.1) under much weaker restrictions which do not imply the absolute convergence of the infinite integrals. For the present, however, it is sufficient to restrict attention to functions $g(t)$ which vanish outside of a finite interval $(a, N)$, where $0<a<N$. Concerning such functions we can use the asymptotic expansion (3.1) to prove the following theorem.

Theorem 9.1. Suppose that (i) $0<a<x<N$, (ii) $g(t)$ is in $L(a, N)$, (iii) $g(t)$ is of bounded variation in some neighborhood of the point $x$, and (iv) $\nu$ is in $V\left({ }^{4}\right)$. Then we have that

$$
\frac{1}{2}\{g(x+)+g(x-)\}=\int_{0}^{\rightarrow \infty} r J_{\nu}(x r) d r \int_{a}^{N} t g(t) J_{\nu}(t r) d t .
$$

An attempt was made in [2, pp. 96-97] to prove the validity of equation (9.2) when (i) and (iv) are true and $g(t)$ satisfies Dirichlet's conditions on $(a, N)$. The proof there given seems incomplete because of a failure to discuss

(4) See the paragraph just following Lemma 2.3 for the definition of $V$. 
adequately the remainder terms in the asymptotic expansion for $J_{\nu}(u)$. Watson [9, p. 464] has made a similar criticism of the original proof of Hankel.

Let $\phi(x)$ stand for the right-hand side of equation (9.2). Then

$$
\begin{aligned}
\phi(x) & =\lim _{h=\infty} \int_{0}^{h} r J_{\nu}(x r) d r \int_{a}^{N} \operatorname{tg}(t) J_{\nu}(t r) d t \\
& =\lim _{h=\infty} \int_{a}^{N} \operatorname{tg}(t) d t \int_{0}^{h} r J_{\nu}(x r) J_{\nu}(t r) d r .
\end{aligned}
$$

When $\nu$ is in $V$ we have from $[9$, p. 134] that

$$
\phi(x)=\lim _{h=\infty} \int_{a}^{N}\left(x^{2}-t^{2}\right)^{-1} \operatorname{tg}(t) K_{0}(x, t, h) d t,
$$

in which $K_{0}(x, t, h)$ is defined as follows:

$$
K_{0}(x, t, h)=h\left\{x J_{\nu+1}(x h) J_{\nu}(t h)-t J_{\nu}(x h) J_{\nu+1}(t h)\right\} .
$$

We have from equations (3.1) that

$$
\begin{aligned}
J_{\nu}(u) & =(2 / \pi u)^{1 / 2} \cos \left(u-\delta_{\nu}\right)+R_{\nu}(u ; 2), \\
R_{\nu}(u ; 2) & =-\left(4 \nu^{2}-1\right)\left(32 \pi u^{3}\right)^{-1 / 2} \sin \left(u-\delta_{\nu}\right)+R_{\nu}(u ; 3) .
\end{aligned}
$$

Hence we have that $K_{0}(x, t, h)=\sum_{i=1}^{5} K_{i}(x, t, h)$, in which $K_{i}(x, t, h)$ are defined as follows:

$$
\begin{aligned}
& K_{1}=\left\{1 / \pi(x t)^{1 / 2}\right\}\{(t-x) \cos [(x+t) h-\nu \pi]+(x+t) \sin (x-t) h\}, \\
& K_{2}=-\left\{\left(4 \nu^{2}-1\right)\left(x^{2}-t^{2}\right) / 4 \pi h\left(x^{3} t^{3}\right)^{1 / 2}\right\} \sin \left(x h-\delta_{v}\right) \sin \left(t h-\delta_{v}\right) \\
& K_{3}=(2 h / \pi)^{1 / 2}\left\{x^{1 / 2} R_{\nu}(t h ; 3) \sin \left(x h-\delta_{\nu}\right)-t^{1 / 2} R_{\nu}(x h ; 3) \sin \left(t h-\delta_{v}\right)\right\} \\
& K_{4}=(2 h / \pi)^{1 / 2}\left\{x t^{-1 / 2} R_{v+1}(x h ; 3) \cos \left(x h-\delta_{v}\right)-t x^{-1 / 2} R_{\nu+1}(t h ; 3) \cos \left(t h-\delta_{v}\right)\right\} \\
& K_{5}=h\left\{x R_{\nu+1}(x h ; 2) R_{\nu}(t h ; 2)-t R_{\nu}(x h ; 2) R_{\nu+1}(t h ; 2)\right\}
\end{aligned}
$$

Let us define $\phi_{i}(x, h)$ as follows:

$$
\phi_{i}(x, h)=\int_{a}^{N}\left(x^{2}-t^{2}\right)^{-1} \operatorname{tg}(t) K_{i}(x, t, h) d t .
$$

Then it is clear that

$$
\begin{aligned}
\phi_{1}(x, h)= & -\pi^{-1} \int_{a}^{N}(t+x)^{-1}(t / x)^{1 / 2} g(t) \cos [(x+t) h-\nu \pi] d \\
& +\pi^{-1} \int_{a}^{N}(t / x)^{1 / 2} g(t)(x-t)^{-1} \sin (x-t) h d t
\end{aligned}
$$

Since $g(t)$ satisfies conditions (i), (ii) and (iii) it follows from well known 
results $\left[7\right.$, pp. 11, 25] that $\lim _{h=\infty} \phi_{1}(x, h)=2^{-1}\{g(x+)+g(x-)\}$. Moreover,

$$
\begin{aligned}
\phi_{2}(x, h) & =-\left\{\left(4 \nu^{2}-1\right) / 4 \pi h x^{3 / 2}\right\} \int_{a}^{N} t^{-1 / 2} g(t) \sin \left(x h-\delta_{\nu}\right) \sin \left(t h-\delta_{v}\right) d t \\
& =o\left(h^{-1}\right) .
\end{aligned}
$$

We shall show that $\phi_{i}(x, h)=O\left(h^{-1}\right)$ when $i=3,4$ or 5 , and this will be sufficient to prove the theorem.

Consider first $K_{3}(x, t, h)$. Using the mean value theorem we see that

$$
\begin{aligned}
\left(\frac{1}{2} \pi / h\right)^{1 / 2} K_{3}(x, t, h) /(x-t)= & \frac{1}{2} y^{-1 / 2} R_{\nu}(t h ; 3) \sin \left(y h-\delta_{\nu}\right) \\
& +h y^{1 / 2} R_{\nu}(t h ; 3) \cos \left(y h-\delta_{\nu}\right) \\
& -h t^{1 / 2} R_{\nu}^{\prime}(y h ; 3) \sin \left(t h-\delta_{v}\right),
\end{aligned}
$$

where $y$ is between $x$ and $t$. As a consequence of Lemma 3.3, the right-hand side is $O\left(h^{-3 / 2}\right)$ uniformly in $x$ and $t$ when $0<a \leqq x, t \leqq N$. Hence,

$$
\phi_{3}(x, h)=\int_{a}^{N}(x+t)^{-1} \operatorname{tg}(t) O\left(h^{-1}\right) d t=O\left(h^{-1}\right) .
$$

It is plain that a similar use of the mean value theorem and of Lemma 3.3 is sufficient to prove that $\phi_{4}(x, h)=O\left(h^{-1}\right), \phi_{5}(x, h)=O\left(h^{-1}\right)$.

10. Proof of Theorem 1.3. Suppose that $x>0$, that $\nu$ is a real number

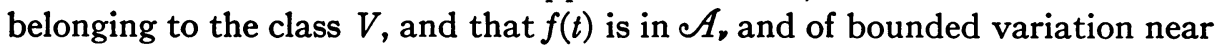
the point $x$. Choose $a$ and $N$ arbitrarily so that $a<x<N$ and define $f(N, a, t)$ to be $f(t)$ if $a \leqq t \leqq N$ and zero otherwise. It is clear that $f(N, a, t)$ is in $\mathcal{A}_{\nu}$ if $f(t)$ is, and we conclude from Lemma 6.1 that if

$$
s_{\nu}(N, a, x)=\sum_{n=0}^{\infty} 2(\nu+2 n+1) J_{\nu+2 n+1}(x) \int_{0}^{\rightarrow \infty} t^{-1} f(N, a, t) J_{\nu+2 n+1}(t) d t
$$

is the Neumann series expansion for $f(N, a, t)$, then

$$
s_{\nu}(N, a, x)=\int_{0}^{\rightarrow \infty} t^{-1} f(N, a, t) \phi_{\nu}(x, t) d t,
$$

whence it follows from equation (2.10) that

$$
s_{\nu}(N, a, x)=x \int_{0}^{\rightarrow \infty} f(N, a, t) d t \int_{0}^{1} J_{\nu}(x r) J_{\nu}(t r) r d r .
$$

Since $f(N, a, t)=0$ when $t>N$ we may invert the order of integration and find that 


$$
\begin{aligned}
s_{\nu}(N, a, x)= & x \int_{0}^{1} r J_{\nu}(x r) d r \int_{a}^{N} f(t) J_{\nu}(t r) d t, \\
s_{\nu}(N, a, x)= & x \int_{0}^{\rightarrow \infty} r J_{\nu}(x r) d r \int_{a}^{N} f(t) J_{\nu}(t r) d t \\
& -x \int_{1}^{\rightarrow \infty} r J_{\nu}(x r) d r \int_{a}^{N} f(t) J_{\nu}(t r) d t .
\end{aligned}
$$

Since $g(t)=t^{-1} f(t)$ satisfies the conditions of Theorem 9.1, we have that

$$
s_{\nu}(N, a, x)=\frac{1}{2}\{f(x+)+f(x-)\}-x \int_{1}^{\rightarrow \infty} r J_{\nu}(x r) d r \int_{a}^{N} f(t) J_{\nu}(t r) d t .
$$

Therefore, Theorem 1.3 is true since it follows from equations (6.7) and (6.8) that $\lim _{N=\infty, a=0} s_{\nu}(N, q, x)=s_{\nu}(x)$ when $\nu$ is real.

11. Further results. We wish now to investigate the possibility of passing to the limit as $N$ approaches $\infty$ and $a$ approaches 0 under the integral sign in equation (1.9). We shall prove the following theorem.

TheOREM 11.1. Suppose that $t^{-1 / 2} f(t)(1+t)^{-1}$ is in $L(0, \infty)$, that $\nu \geqq-1 / 2$, and that the integral

$$
\int_{0}^{\rightarrow \infty} f(t) J_{\nu}(t r) d t
$$

converges uniformly in $r$ on any interval interior to $(1, \infty)$. Then we have that

$$
s_{\nu}(x)=\frac{1}{2}\{f(x+)+f(x-)\}-x \int_{1}^{\rightarrow \infty} r J_{\nu}(x r) d r \int_{0}^{\rightarrow \infty} f(t) J_{\nu}(t r) d t .
$$

It is worth noticing that the hypotheses of Theorem 11.1 will surely hold if $t^{-1 / 2} f(t)$ is in $L(0, \infty)$. When $\nu \geqq-1 / 2$, the function $f(t)$ is surely in $A_{v}$. It follows that in order to prove Theorem 11.1 it is sufficient to show that

$$
\lim _{N=\infty, a=0} \int_{1}^{\rightarrow \infty} r J_{\nu}(x r) d r\left(\int_{0}^{a}+\int_{N}^{\rightarrow \infty}\right) f(t) J_{\nu}(t r) d t=0 .
$$

Choose numbers $a, b$ and $c$ such that $1<b<c, 0<a \leqq x / 2$. Then

$$
\int_{b}^{c} r J_{\nu}(x r) d r \int_{0}^{a} f(t) J_{\nu}(t r) d t=\int_{0}^{a} f(t) d t \int_{b}^{c} r J_{\nu}(x r) J_{\nu}(t r) d r .
$$

The inner integral on the right has the value $[9, \mathrm{p} .134]$

$$
\left.\left(x^{2}-t^{2}\right)^{-1}\left\{r x J_{\nu+1}(x r) J_{\nu}(t r)-r t J_{\nu}(x r) J_{\nu+1}(t r)\right\}\right|_{r=b} ^{r=c} \text {. }
$$


Since $t \leqq a \leqq x / 2$, we have that $0 \leqq\left(x^{2}-t^{2}\right)^{-1} \leqq 4 / 3 x^{2}$. Moreover since $\nu \geqq-1 / 2$, there exists a constant $B_{\nu}$ such that $u^{1 / 2}\left|J_{\nu}(u)\right| \leqq B_{\nu}, u^{1 / 2}\left|J_{\nu+1}(u)\right| \leqq B_{\nu}$. Therefore, the expression (11.5) is dominated by $\left(8 B_{\nu}^{2} / 3 x^{2}\right)\left(x^{1 / 2} t^{-1 / 2}+x^{1 / 2} t^{1 / 2}\right)$ $\leqq 4 B_{\nu}^{2}\left(x^{3} t\right)^{-1 / 2}$, and the expressions (11.4) are dominated by

$$
4 B_{\nu}^{2} x^{-3 / 2} \int_{0}^{a} t^{-1 / 2}|f(t)| d t
$$

If we let $b$ approach one and $c$ approach $\infty$, and then let $a$ approach zero, we conclude that

$$
\lim _{a=0} \int_{1}^{\rightarrow \infty} r J_{\nu}(x r) d r \int_{0}^{a} f(t) J_{\nu}(t r) d t=0 .
$$

Now choose numbers $b, c, N$ and $P$ such that $1<b<c, 2 x \leqq N<P$. Then

$$
\int_{b}^{c} r J_{\nu}(x r) d r \int_{N}^{P} f(t) J_{\nu}(t r) d t=\int_{N}^{P} f(t) d t \int_{b}^{c} r J_{\nu}(x r) J_{\nu}(t r) d r .
$$

Since $t \geqq N \geqq 2 x$, we have that $0 \leqq\left(t^{2}-x^{2}\right)^{-1} \leqq 4 / 3 t^{2}$. Reasoning as in the preceding paragraph, we obtain upon letting $P$ approach $\infty$ that

$$
\left|\int_{N}^{\rightarrow \infty} f(t) d t \int_{b}^{c} r J_{\nu}(x r) J_{\nu}(t r) d r\right| \leqq 4 B_{\nu}^{2} x^{-1 / 2} \int_{N}^{\infty} t^{-3 / 2}|f(t)| d t .
$$

For each choice of $b, c, N$ and a positive quantity $\epsilon$, we may select $P$ so that $P>N$ and that

$$
\begin{aligned}
& \left|r J_{\nu}(x r) \int_{P}^{\rightarrow \infty} f(t) J_{\nu}(t r) d t\right|<\epsilon / 2 c \quad(b \leqq r \leqq c), \\
& 4 B_{\nu}^{2} x^{-1 / 2} \int_{P}^{\infty} t^{-3 / 2}|f(t)| d t<\epsilon / 2 .
\end{aligned}
$$

It now follows from equation (11.7) and the inequality (11.8) that

$$
\begin{aligned}
& \left|\int_{b}^{c} r J_{\nu}(x r) d r \int_{N}^{\rightarrow \infty} f(t) J_{\nu}(t r) d t-\int_{N}^{\rightarrow \infty} f(t) d t \int_{b}^{c} r J_{\nu}(x r) J_{\nu}(t r) d r\right| \\
& \quad \leqq\left|\int_{P}^{\rightarrow \infty} f(t) d t \int_{b_{v}}^{c} r J_{\nu}(x r) J_{\nu}(t r) d r\right|+\int_{b}^{c}\left|r J_{\nu}(x r) d r \int_{P}^{\rightarrow \infty} f(t) J_{\nu}(t r) d t\right|<\epsilon .
\end{aligned}
$$

If we let $\epsilon$ approach zero and use the inequality (11.8) we find that

$$
\left|\int_{b}^{c} r J_{\nu}(x r) d r \int_{N}^{\rightarrow \infty} f(t) J_{\nu}(t r) d t\right| \leqq 4 B_{\nu}^{2} x^{-1 / 2} \int_{N}^{\infty} t^{-3 / 2}|f(t)| d t .
$$

If we let $b$ approach one and $c$ approach $\infty$, and then let $N$ approach $\infty$, we 
can conclude that

$$
\lim _{N=\infty} \int_{1}^{\rightarrow \infty} r J_{\nu}(x r) d r \int_{N}^{\rightarrow \infty} f(t) J_{\nu}(t r) d t=0 .
$$

Equation (11.3) now follows from equations (11.6) and (11.9), and this completes the proof of Theorem 11.1.

The methods used in proving Theorem 11.1 may also be used to prove the following version of the Hankel formula.

Theorem 11.2. Suppose that (i) $x>0$, (ii) $t^{1 / 2} g(t)(1+t)^{-1}$ is in $L(0, \infty)$, (iii) $g(t)$ is of bounded variation in some neighborhood of the point $x$, (iv) $R(\nu)>-1 / 2$, and $(\mathrm{v})$ the integral

$$
\int_{0}^{\rightarrow \infty} \operatorname{tg}(t) J_{\nu}(t r) d t
$$

converges uniformly in $r$ on any interval interior to $(0, \infty)$. Then we have that

$$
\frac{1}{2}\{g(x+)+g(x-)\}=\int_{0}^{\rightarrow \infty} r J_{\nu}(x r) d r \int_{0}^{\rightarrow \infty} t g(t) J_{\nu}(t r) d t .
$$

Let $f(t)=\operatorname{tg}(t)$. It follows from Theorem 9.1 that if $a<x<N$ we have that

$$
\frac{1}{2}\{f(x+)+f(x-)\}=x \int_{0}^{\rightarrow \infty} r J_{\nu}(x r) d r \int_{a}^{N} f(t) J_{\nu}(t r) d t
$$

so that Theorem 11.2 is valid if its hypotheses imply that equation (11.3) holds when the interval of integration $(1, \infty)$ is replaced by $(0, \infty)$. The proof of this result is identical with that of equation (11.3) except that $b$ is now made to approach 0 instead of 1 . The fact that $\nu$ may be complex causes no difficulty.

12. Combination of two expansions. Suppose that $f(x)$ is neither even nor odd. Then it is natural to attempt to expand it into a series of the form

$$
\sum_{n=0}^{\infty} a_{n} J_{n}(x)
$$

in which Bessel functions of all integral orders occur. Such a series may be formally obtained by splitting $f(x)$ into an even and an odd part, say $f(x)=g(x)+h(x)$, and expanding $g(x)$ and $h(x)$ into series of even and odd Bessel functions respectively. We find from Theorem 1.2 that if $g(t)$ is in $A_{-1}$ and is absolutely continuous on $(0, x)$, then

$$
s_{-1}(x)=g(x-)-g(0+) J_{0}(x)-\int_{0}^{x} J_{0}(x-v) G(v) d v,
$$


where $G(v)$ is defined by equation (1.3) with $f(t)$ replaced by $g(t)$. Similarly, if $h(t)$ is in $A_{0}$ and is absolutely continuous on $(0, x)$, then

$$
s_{0}(x)=h(x-)-h(0+) J_{0}(x)-\int_{0}^{x} J_{0}(x-v) H(v) d v,
$$

where $H(v)$ is defined by equation (1.3) with $f(t)$ replaced by $h(t)$. Adding these last two results shows that

$$
s_{0}(x)+s_{-1}(x)=f(x-)-f(0+) J_{0}(x)-\int_{0}^{x} J_{0}(x-v) F(v) d v
$$

The left-hand side of equation (12.2) may be written in the form

$$
s(x)=\sum_{n=0}^{\infty} 2 n J_{n}(x) \int_{\rightarrow-\infty}^{\rightarrow \infty}|t|^{-1} J_{n}(t) f(t) d t,
$$

if one remembers that $g(x)=\{f(x)+f(-x)\} / 2, h(x)=\{f(x)-f(-x)\} / 2$. We may now prove the following theorem.

Theorem 12.1. Suppose that $t^{-1} f(t)(1+|t|)^{1 / 2-p}$ is in $L(-\infty, \infty)$ for some nonnegative integer $p$, and that the limits

$$
\int_{a}^{\rightarrow \infty} e^{i t} f( \pm t) t^{-3 / 2} d t
$$

exist when $a>0$. Then if $x>0$ and $f(t)$ is absolutely continuous on the interval $(-x, x)$, we have that

$$
s(x)=f(x-)-\int_{0}^{x} J_{0}(x-v) F(v) d v,
$$

while if $x<0$, the term $f(x-)$ is replaced by $f(x+)$.

For the integrability hypotheses imply that $g(t)$ is in $\mathcal{A}_{-1}$ and that $h(t)$ is in $A_{0}$. Moreover, since $f(t)$ is continuous at the origin and $t^{-1} f(t)$ is integrable in a neighborhood of the origin, it must be true that $f(0)=0$. The theorem when $x>0$ now follows from equation (12.2). We deduce the result when $x<0$ from the result when $x>0$ by considering the function $f(-t)$.

13. Some examples. The function used by the early writers to show that it was not true that $s_{0}(x)=f(x)$ for all functions $f(t)$ was $\sin \alpha t$. If $0 \leqq \alpha \leqq 1$ and $\nu=0$ this function satisfies the hypotheses of Theorem 11.1 and the integral (11.1) has the value zero $\left[9\right.$, p. 405]. Hence the Neumann series $s_{0}(x)$ converges to $\sin \alpha x$ when $0 \leqq \alpha \leqq 1$. If $\alpha>1$ the integral (11.1) is not uniformly convergent on an arbitrary interval $(b, c)$ interior to $(1, \infty)$, the point $r=\alpha$ being exceptional. The integral is, however, uniformly convergent to 0 on any interval interior to $(\alpha, \infty)$ and to $\left(\alpha^{2}-r^{2}\right)^{-1 / 2}$ on any interval interior to 
$(1, \alpha)[9$, p. 405]. It is easy to see that Theorem 11.1 remains true under these circumstances. When $\alpha>1$ we therefore have that

$$
s_{0}(x)=\sin \alpha x-x \int_{1}^{\alpha} r J_{0}(x r)\left(\alpha^{2}-r^{2}\right)^{-1 / 2} d r .
$$

We may also conclude from Theorem 1.2 and $[9$, p. 405] that

$$
s_{0}(x)=\sin \alpha x-\left(\alpha^{2}-1\right)^{1 / 2} \int_{0}^{x} J_{0}(x-v) \cos \alpha v d v
$$

when $\alpha>1$.

Another example when $\nu=0$ is $f(t)=t^{-1}\left\{1-J_{0}(\alpha t)\right\}$. This function also satisfies the hypotheses of Theorem 11.1. In fact, $t^{-1 / 2}|f(t)|$ is in $L(0, \infty)$. By [9, p. 406] the integral (11.1) has the value 0 if $r \geqq \alpha$ and $\log (\alpha / r)$ if $r \leqq \alpha$. Therefore, the Neumann series $s_{0}(x)$ converges to $f(x)$ if $\alpha \leqq 1$, while if $\alpha>1$ it converges to

$x^{-1}\left\{1-J_{0}(\alpha x)\right\}+x \int_{1}^{\alpha} r \log (r / \alpha) J_{0}(x r) d r=x^{-1}\left\{1-J_{0}(x)+x J_{1}(x) \log \alpha\right\}$.

\section{BIBLIOGRAPHY}

1. H. Bateman, On an expansion of an arbitrary function in a series of Bessel functions, Messenger of Mathematics vol. 36 (1907) pp. 31-37.

2. A. Gray, G. B. Mathews, and T. M. MacRobert, A treatise on Bessel functions, MacMillan, 1931.

3. W. Kapteyn, On a series of Bessel functions, Koninklijke Akademie van Wetenschappen te Amsterdam, Proceedings of the Section of Sciences, vol. 7 (1905) pp. 494-500.

4. - On an expansion of an arbitrary function in a series of Bessel functions, Messenger of Mathematics vol. 35 (1906) pp. 122-125.

5. W. V. Lovitt, Linear integral equations, McGraw-Hill, 1924.

6. S. Pincherle, Sopra alcuni sviluppi in serie per funzioni analitiche, R. Accademia delle scienze dell'Istituto di Bologna, Memoria (4) vol. 3 (1881) pp. 151-180.

7. E. C. Titchmarsh, Introduction to the theory of Fourier integrals, Oxford University Press, 1939.

8. - The theory of functions, Oxford University Press, 1939. 1945.

9. G. N. Watson, A treatise on the theory of Bessel functions, Cambridge University Press,

10. H. A. Webb, The expansion of an arbitrary function in a series of Bessel functions, Messenger of Mathematics vol. 33 (1904) pp. 55-58. 1943.

11. E. T. Whittaker and G. N. Watson, Modern analysis, Cambridge University Press,

\section{American Optical Company,}

BuFfalo, N. Y. 